\title{
14. LATE NEOGENE SILICOFLAGELLATES AND EBRIDIANS FROM LEG 128, SEA OF JAPAN ${ }^{1}$
}

\author{
Hsin-Yi Ling ${ }^{2}$
}

\begin{abstract}
Datum levels and biostratigraphy, including a new zonation of late Neogene silicoflagellates and ebridians, are presented, based on analysis of submarine sediments collected from Sites 798 and 799, Sea of Japan, during the Leg 128 cruise.
\end{abstract}

\section{INTRODUCTION}

Silicoflagellates and ebridians play an important role in biostratigraphy, next only to diatoms in their consistent occurrence in submarine sediments from the Sea of Japan recovered during the Leg 128 cruise of the Ocean Drilling Program (ODP). Furthermore, the detailed shipboard paleomagnetic analysis established the "absolute ages" for their biostratigraphic events.

\section{MATERIALS AND METHODS}

The oceanographic data for Leg 128 (Fig. 1) are Site 798, at $37^{\circ} 03832^{\prime} \mathrm{N}, 134^{\circ} 79976^{\prime \prime} \mathrm{E}$, in $903.1 \mathrm{~m}$ water depth, and Site 799 , at $39^{\circ} 22046^{\prime} \mathrm{N}, 133^{\circ} 86685^{\prime} \mathrm{E}$, in $2073.0 \mathrm{~m}$ water depth.

The procedures for sample preparation and the methods used to designate the location of illustrated specimens in the strewn slides as well as the relative abundance of taxa within each sample are essentially the same as described in the Deep Sea Drilling Project (DSDP) Leg 19 report (Ling, 1973). All microslides used for the present investigation, including the figured specimens, will be deposited permanently in the Micropaleontology Collection of the Department of Geology, Northern Illinois University.

\section{LATE NEOGENE SILICOFLAGELLATE/EBRIDIAN ZONATION FROM THE SEA OF JAPAN}

In this section, the development of silicoflagellate/ebridian zonation from the Sea of Japan is reviewed in conjunction with that of the northwest Pacific, followed by the detailed documentation of datum levels based on recent results and the presentation of a new zonation in the Sea of Japan.

\section{Previous Zonation}

The biostratigraphic zonation of silicoflagellates from the midlatitude North Pacific was proposed by Bukry (1973a) for DSDP Site 173 off California in correlation with the coccolith zones (Fig. 2). After the successful biostratigraphic application of a combination of silicoflagellates and ebridians during DSDP Leg 19, Ling (1973) presented a combined zonation from the high-latitude North Pacific, which he later revised based on the analysis of Leg 31 subsurface sediments, including from the Sea of Japan (Ling, 1975). Age assignments for these zones were made by correlation with co-occurring diatoms (Koizumi, 1975). Further examination of Northwest Pacific sediments revealed that the last appearance datum (LAD) of Dictyocha subarctios occurs slightly above the Brunhes/Matuyama Chron

\footnotetext{
${ }^{1}$ Pisciotto, K. A., Ingle, J. C., Jr., von Breymann, M. T., Barron, J., et al., 1992. Proc. ODP, Sci. Resuits, 127/128, Pt. 1: College Station, TX (Ocean Drilling Program).

${ }^{2}$ Department of Geology, Northern Illinois University, DeKalb, IL 60115, U.S.A.
}

boundary of approximately $0.7 \mathrm{Ma}$ (Ling, 1976). Hays et al. (1969) and Saito and Burckle (1977) reported the last occurrence of Mesocena cf. elliptica between the Brunhes Chron and Jaramillo Subchron, which in the eastern Pacific is at about $0.73 \mathrm{Ma}$.

Barron (1980) incorporated silicoflagellate events into his diatom zonation for DSDP Leg 57 sediments from the Northwest Pacific, correlated them with the paleomagnetic stratigraphy, and listed the estimated "absolute ages." The silicoflagellate datums included are:

LAD Mesocena quadrangula between Olduvai and Jaramillo, $1.5 \mathrm{Ma}$ LAD Distephanus pseudofibula

Overlap of $D$. pseudofibula and Thalassiosira antiqua (diatom)

Overlap of D. pseudofibula and Mesocena hexagona

Later Bukry and Monechi (1985) proposed a silicoflagellate zonation based on DSDP Leg 86 samples, also from the Northwest Pacific (Fig. 2). It should be pointed out that they left most of the Pliocene, the entire late Miocene, and most of the Middle Miocene unzoned. As discussed in the following, these zonal gaps can now be satisfactorily filled for the Sea of Japan by a combination of silicoflagellates and ebridians.

Kobayashi (1988) recognized 11 silicoflagellate datum levels and 12 zones for the Neogene interval from the Japan Sea coastal region and DSDP Hole 438A, located in the northwest corner of the North Pacific. His uppermost three zones, Distephanus jimlingii, Paramesocena circulus, and Dictyocha neopseudofibula in ascending order, which represent the entire Pliocene, can be generally followed during the present study.

\section{Datum Levels}

The datum levels recognized from the Sea of Japan (Fig. 3) are discussed in ascending order. They are grouped in two categories: first order (designated by bold letters), which have been recognized previously and/or correlated with the occurrence of other groups of both calcareous and siliceous microfossils and the estimated "absolute age" based on magnetostratigraphy, including Leg 128 shipboard analysis (Ingle, Suyehiro, von Breymann, et al., 1990); second order (designated by plain letters), which have been observed but require further observation, or the datum has not been correlated with other microfossil data or magnetic reversal history.

\section{First-appearance datum (FAD) of Distephanus jimlingii}

Although Kobayashi (1988) applied this datum level to draw the Miocene-Pliocene boundary, this datum could not be followed due to its inconsistent distribution in the present Leg 128 samples.

\section{FAD of Dictyocha neopseudofibula}

Kobayashi (1988) recognized the datum level as the base of his uppermost (12) zone and the early/late Pliocene boundary. This datum level occurs between Samples 128-798B-34X-5, 30-32 cm, and 


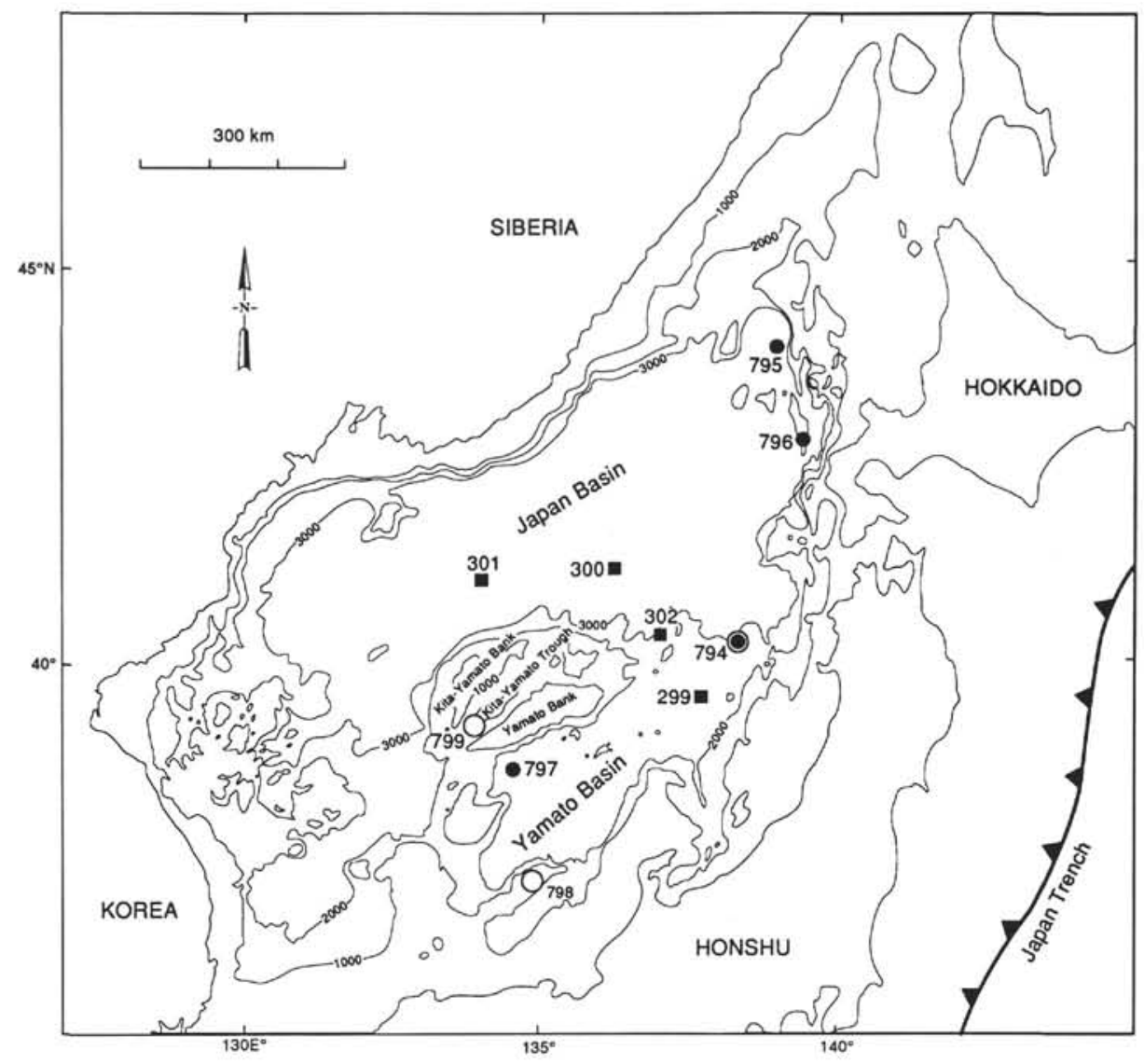

Figure 1. Bathymetry (in meters) of the Sea of Japan. Sites 794, 798, and 799 drilled during Leg 128 are shown by open circles. Sites 299-302 drilled during DSDP Leg 31 are indicated by solid circles, and Sites 794-797 drilled during Leg 127 are shown by solid circles. Site 794 was drilled during both Legs 127 and 128.

128-798B-34X-CC at 321.16-321.95 m below seafloor (mbsf). Furthermore, Kobayashi's observation of the slight upward extension of the geological range of Paramesocena circulus above this datum is in agreement with observation of Hole 798B samples.

This datum would be more reliable if a similar occurrence were observed in other subsurface sections or if the distribution of Paramesocena circulus were more consistent.

\section{Last-appearance datum (LAD) of Distephanus jimlingii}

The LAD of D. jimlingii (as Cannopilus hemisphericus in Ling, 1975 ) was considered to signal the top of the early Pliocene and was correlated with the top of the Denticulopsis seminae var. fossilis-D. kamtschatica (diatom) Zone (Koizumi, 1975; Koizumi and Tanimura, 1985). The datum falls between 128-798B-30X-CC and 128-798B$31 X-1,30-32 \mathrm{~cm}$ (286.40-286.70 mbsf). Because the Matuyama/Gauss Chron boundary, 2.47 Ma, was determined at $297.9 \mathrm{mbsf}$, the $D$. jimlingii datum occurs slightly higher than the chron boundary, but is within late Pliocene.

In Hole 798B, the LAD of Dictyocha neopseudofibula coincides with this datum level.

\section{LAD of Ebriopsis antiqua antiqua}

The top of E. antiqua antiqua was thought to mark the late and early late Pliocene boundary (Ling, 1975) and fall within the Denticula seminae var. fossilis (diatom) Zone (Koizumi, 1975; Koizumi and Tanimura, 1985). In Hole 798B, the datum is observed between Samples 128-798B-30X-3, 30-32 cm, and 128-798B-30X-4, 30-32 $\mathrm{cm}$ (280.5-282.0 mbsf), and the Matuyama/Gauss Chron boundary, $2.47 \mathrm{Ma}$, is considered to be at $297.9 \mathrm{mbsf}$, within Core 128-798B$31 \mathrm{X}$. In Hole $799 \mathrm{~A}$, the datum was recognized between Samples 128-799A-17H-5, $131 \mathrm{~cm}$, and 128-799A-17H-6, 83-85 cm (151.3$153.83 \mathrm{mbsf}$ ), and the Matuyama/Gauss boundary was reported at 174 mbsf in Section 128-799A-19X-4.

\section{LAD of Ammodochium rectangulare}

This datum level was considered to be at approximately the Pliocene/Pleistocene boundary (Ling, 1975). The datum level is located between Samples 128-798B-20X-CC and 128-798B-21X-11 $50-52 \mathrm{~cm}$ (191-191.5 mbsf), whereas the top of the Olduvai Subchron $(1.66 \mathrm{Ma})$ is considered to be at $200 \mathrm{mbsf}$ within Section 128-798B-21X-7. In Hole 799A, the datum level is placed between 128-799A-15H-4, 76-78 cm, and 128-799A-15H-5, 76-78 cm (131.36$132.86 \mathrm{mbsf}$ ), and the top of the Olduvai Subchron is placed at 123 mbsf, which is within Section 128-799A-14H-5. Thus, analysis from the Sea of Japan further confirms that the datum approximates the Pliocene/Pleistocene boundary but is slightly diachronous between Holes 798B and 799A.

\section{LAD of Dictyocha subarctios}

The top of Dictyocha subarctios was considered slightly above the Brunhes/Matuyama Chron boundary and near the top of the Nitzchia reinboldii (diatom) Zone in the North Pacific (Ling, 1976). In Hole 798A, the datum is observed between Samples 128-798A$9 \mathrm{H}-1,32-34 \mathrm{~cm}$, and $128-798 \mathrm{~A}-8 \mathrm{H}-\mathrm{CC}$ (74.8-75.12 mbsf), whereas 


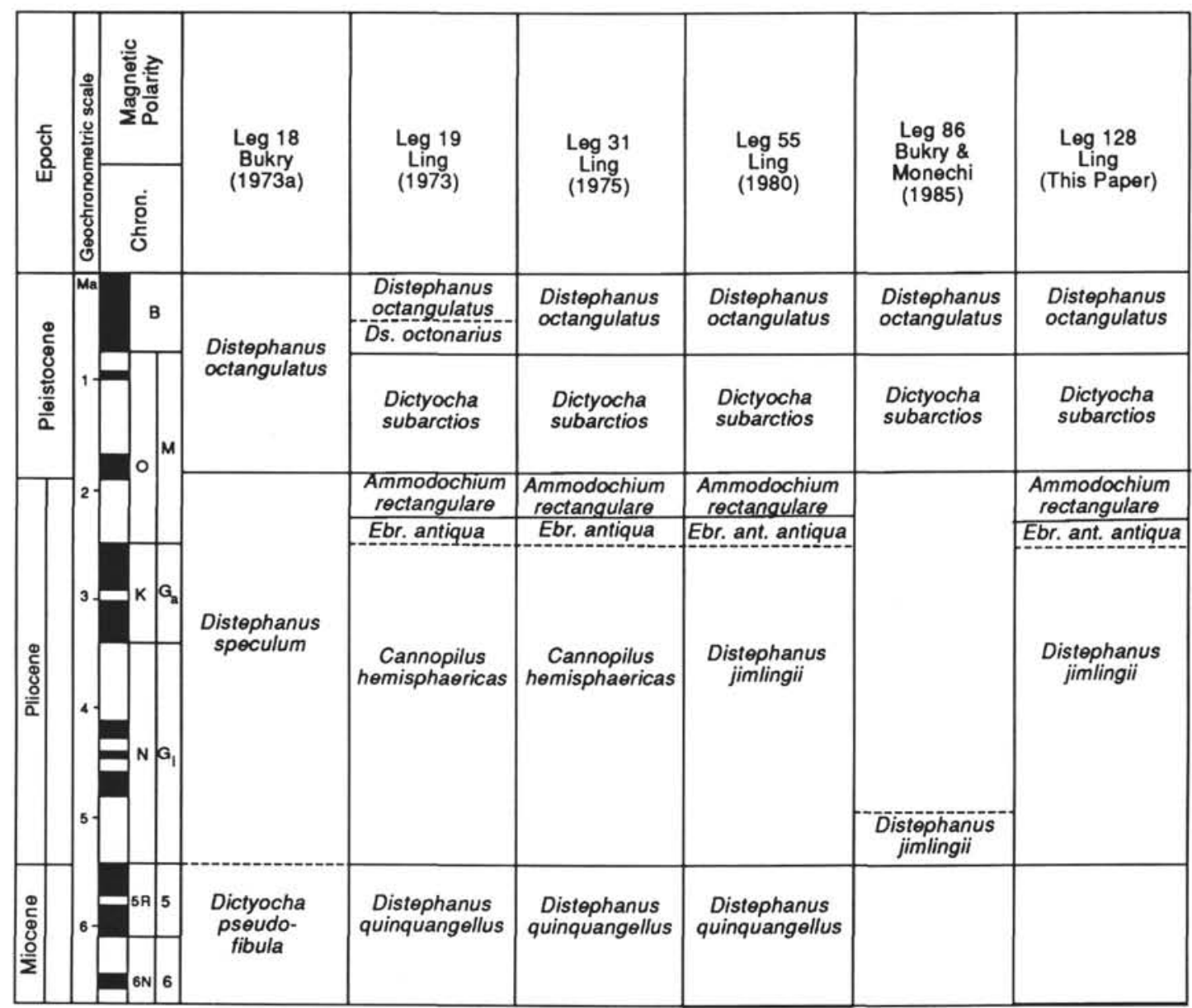

Figure 2. Silicoflagellate and ebridian zonation in the northwest Pacific and the Sea of Japan.

the Brunhes/Matuyama boundary, $0.73 \mathrm{Ma}$, is placed at $75.34 \mathrm{mbsf}$, within Section 128-798A-9H-1. On the other hand, in Hole 799A, the datum 3 is recognized between Samples 28-799A-11 H-1, $115 \mathrm{~cm}$, and $128-799 \mathrm{~A}-11 \mathrm{H}-2,29 \mathrm{~cm}(88.65-89.25 \mathrm{mbsf})$ whereas the Brunhes/Matuyama boundary is placed at $73 \mathrm{~m}$ (Ingle, Suyehiro, von Breymann, et al., 1990) (Fig. 2). ${ }^{3}$

As mentioned in the preceding review, the LAD of Mesocena elliptica was considered to occur between the base of the Brunhes Chron and the top of the Jaramillo Subchron in the equatorial Pacific (Hays et al., 1969) and on the Oga Peninsula, in the western part of northeast Japan (Saito and Burckle, 1977). However, Bukry and Monechi (1985) (as M. quadrangula) considered them as nearly correlative with the present $D$. subarctios datum.

\section{LAD of Distephanus crux var. stauracanthus}

The FAD of this species is either slightly before or after the LAD of Dictyocha subarctios. However, its LAD is always above or younger than the LAD of D. subarctios in the Central North Pacific (Ling, 1970) or high-latitude North Pacific (Ling, 1973), and thus is within the Brunhes Chron. Examination of additional samples should define the datum more closely.

\footnotetext{
${ }^{3}$ Samples with a specific depth rather than an interval are smear slides that were prepared by shipboard sedimentologists for lithologic description and examined by the present author during the cruise.
}

\section{NEW SILICOFLAGELLATE AND EBRIDIAN ZONATION FROM THE SEA OF JAPAN}

Based on the preceding datum levels, the following new zonation is proposed for the northwestern Pacific, including the Sea of Japan. They are discussed in ascending order:

\section{Distephanus jimlingii Zone}

Age: early to early late Pliocene

Top: LAD of Distephanus jimlingii

Base: FAD of Distephanus jimlingii

Remarks: Kobayashi (1988) recognized the Paramesocena circulus Interval Zone, prior to the FAD of Dictyocha neopseudofibula. However, during the present study, the occurrence of Paramesocena circulus was too limited, only in samples from Cores 128-798B-33X and 128-798B-34X, to identify Kobayashi's zone. Kobayashi (1988) also established the Dictyocha neopseudofibula Zone with the FAD of $D$. neopseudofibula as the base of the zone. Because the occurrence of $D$. neopseudofibula was observed only in Hole 798B samples, the author will not follow Kobayashi's practice until further analysis from the area including Leg 127 samples is completed.

The top of this zone is defined by the LAD of Distephanus jimlingii because of its occurrence in samples of both holes. It should be noted that in Hole 798B this datum also coincides with that of Dictyocha neopseudofibula.

\section{Ebriopsis antiqua antiqua Zone}

Age: late late Pliocene

Top: LAD of Ebriopsis antiqua antiqua

Bottom: LAD of Distephanus jimlingii 
798A

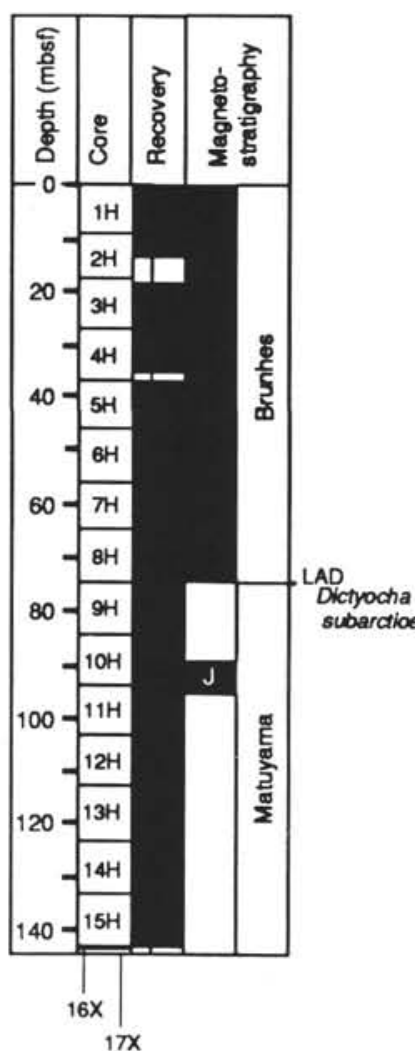

$798 \mathrm{C}$

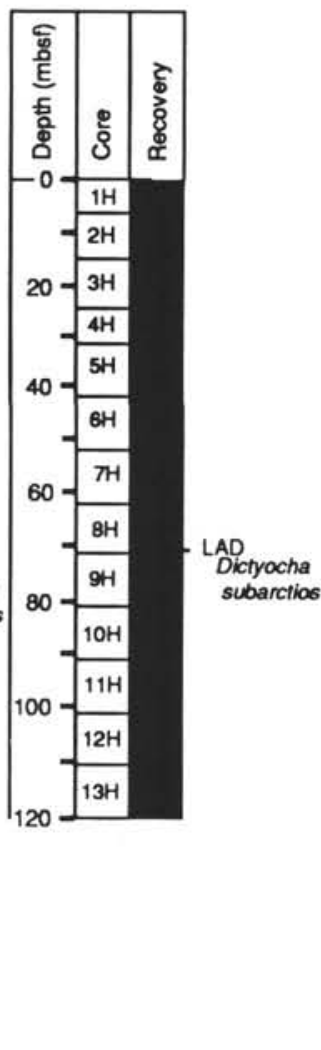

798B

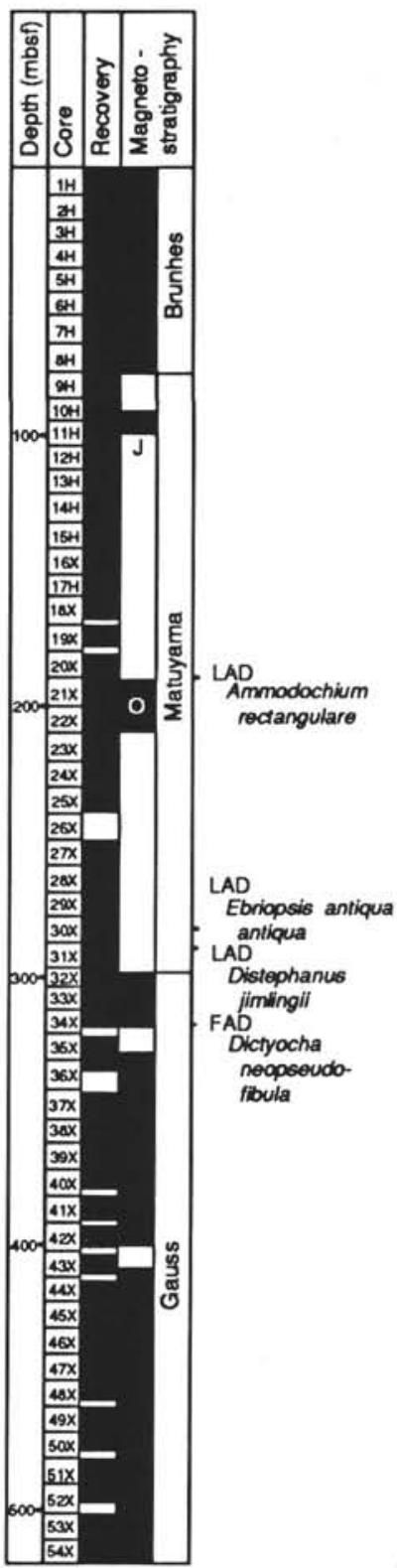

799A

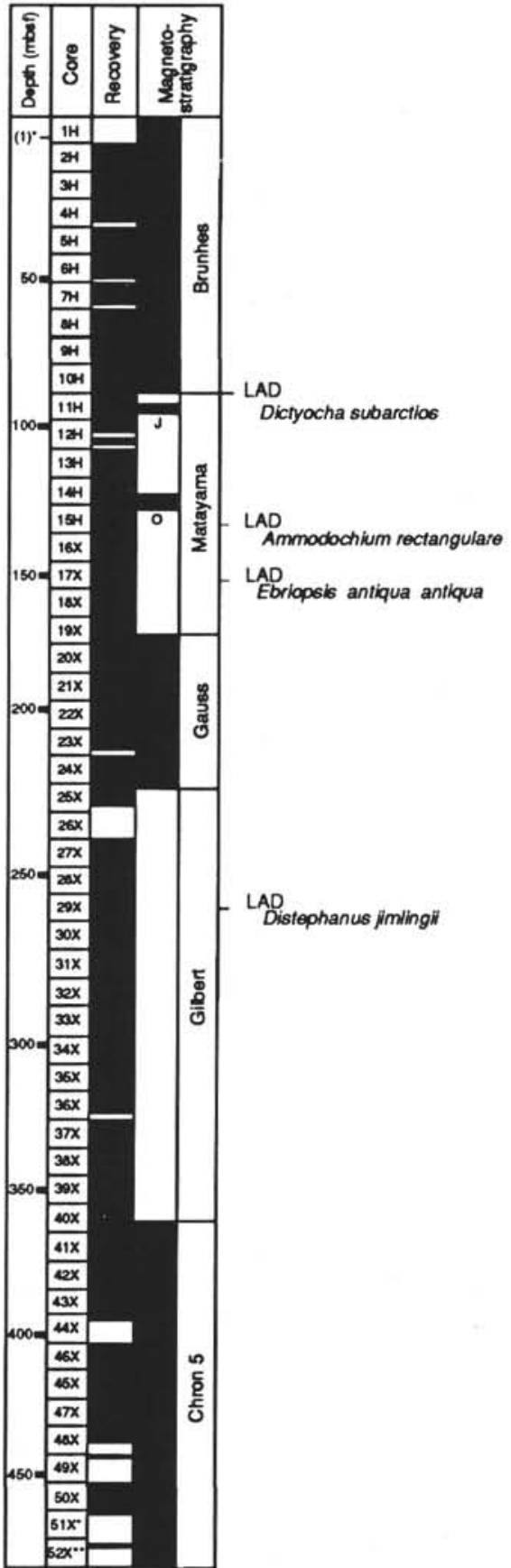

Figure 3. Core recovery, magnetostratigraphy, and silicoflagellate and ebridian datum levels from Sites 798 and 799 . FAD = first appearance datum; LAD $=$ last appearance datum.

Remarks: Except for the nomenclature of the index species, which was originally called either $E$. antiqua (spineless form, Ling, 1973) or E. antiqua (without spine form, Ling, 1975), the original definition for the zone remains the same. In comparison with the results of paleomagnetic analysis, the top of this zone is slightly older than the Matuyama/Gauss Chron boundary of $2.47 \mathrm{Ma}$.

Ammodochium rectangulare Zone

Age: late late Pliocene
Top: LAD of Ammodochium rectangulare

Base: coincides with the top of the underlying Ebriopsis antiqua antiqua Zone.

Remarks: As discussed previously, the top of this zone nearly corresponds to the Pliocene/Pleistocene boundary.

\section{Dictyocha subarctios Zone}

Age: early Pleistocene

Top: LAD of Dictyocha subarctios. 
Base: top of the underlying Ammodochium rectangulare Zone

Remarks: The original definition for the zone remains correct. Bukry and Monechi (1985) favored using the local range of the named species as an alternative criterion for the zone. However, their definition is not acceptable for the following reasons. First, the initial appearance of $D$. subarctios is slightly above the Pliocene/Pleistocene boundary. Therefore, by adopting its local range for the zone, there will be a time gap between the Pliocene/Pleistocene boundary and the initial appearance of the species. Second, as documented previously, the LAD of Ammodochium rectangulare has been observed at several sites, readily recognizable and considered to be a good boundary marker.

LAD of Mesocena quadrangula is thought to correspond to the top of this zone. For practical purposes, this assumption is correct. However, the datum is actually observed slightly higher than that of Dictyocha subarctios.

\section{Distephanus octangulatus Zone}

Age: late Pleistocene to Holocene

Top: Recent

Base: top of the underlying Dictyocha subarctios Zone

Remarks: The zone encompasses the Distephanus octangulatus and D. octonarius Zones of the high-latitude North Pacific (Ling, 1973). The rare occurrence of $D$. octonarius in the Japan Sea sediments makes it necessary to combine these two zones into one.

As stated previously, the LAD of Dictyocha subarctios is observed slightly above the Brunhes/Matuyama Chron boundary.

\section{SITE SUMMARIES}

Throughout the shipboard and subsequent shorebased analyses of submarine samples, the occurrence of silicoflagellates and ebridians is consistent, and their abundance is surpassed only by that of diatoms.

\section{Site 798}

Moderately well preserved, rare to common silicoflagellates and ebridians were observed continuously in cored sediments of Holes $798 \mathrm{~A}, 798 \mathrm{~B}$, and 798C (Tables 1 through 3, respectively) except in the deepest seven basal cores (128-798B-48X to 128-798B-54X; 450.4-517.9 mbsf), which were barren of these microfossils.

Based on the datum levels and the proposed zonation, correlation among them can be summarized as follows:

The LAD of Dictyocha subarctios was observed in Sample 128$798 \mathrm{~A}-9 \mathrm{H}-1,32-34 \mathrm{~cm}$ (75.12 mbsf), marking the top of the $D$. subarctios Zone, with the overlying section assigned to the Distephanus octangulatus Zone. The Dictyocha subarctios Zone continues to the bottom of the hole (Section 128-798A-15H-CC, 142.5 mbsf).

Examination of samples from Hole 798B started at Section 128798B-14H-CC, in order to avoid duplicating the uppermost interval, and continued to the bottom of the hole.

The LAD of Ammodochium rectangulare was encountered in Sample 128-798B-21X-1, 50-52 cm (191.5 mbsf), marking the upper limit for the zone of the named species, indicating the Pliocene/Pleistocene boundary. The LAD of Ebriopsis antiqua antiqua was recognized in Sample 128-798B-30X-4, 30-32 cm ( $282.0 \mathrm{mbsf}$ ), where the top of the E. antiqua antiqua Zone is drawn. The LAD of Distephanus jimlingii was observed in Sample 128-798B-31X-1, 30-32 cm (286.70 mbsf), which defines the top of the zone of the named species. The zone extends downward to Sample 128-794B-48X-2, 25-27 cm (452.15 mbsf), the last silicoflagellate-bearing sample from this hole.

In the Hole $798 \mathrm{C}$ sediments, the LAD of Dictyocha subarctios was observed in Sample 128-798C-9H-1, 20 cm (972.1 mbsf), indicating the top of the D. subarctios Zone, and the overlying section is assigned to the Distephanus octangulatus Zone. The occurrence of Dictyocha subarctios continues down to the deepest sample of the hole, Sample 128-798C-13H-CC (120.1 mbsf), suggesting that the sediments are still in the $D$. subarctios Zone.
Table 1. Distribution of silicoflagellates and ebridians from Hole 298A.

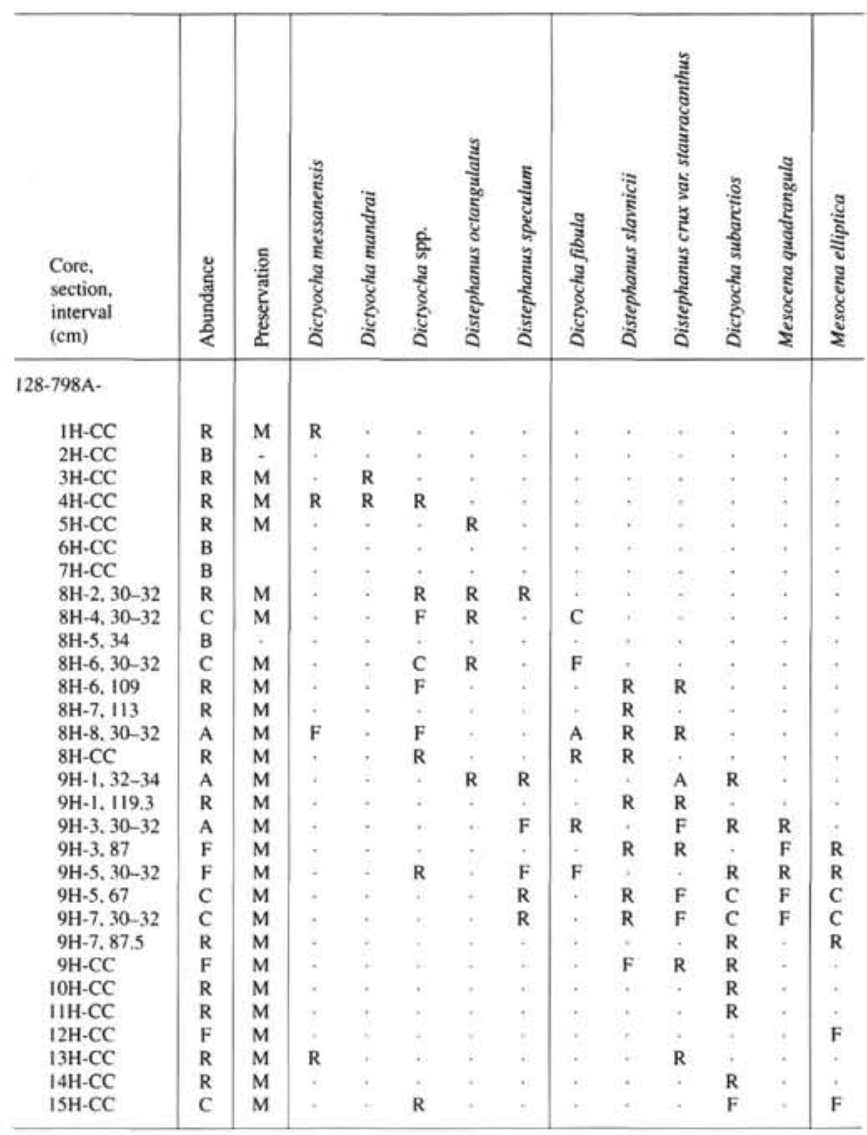

Abundance: A, abundant: C. common: F. few: R. rare: and B. barren. Preservation: G. good: M. moderate: and P. poor

\section{Site 799}

Silicoflagellates and ebridians were observed in most of the samples examined from Hole 799A (Table 4), but their abundance is generally lower than that of Site 798. Only rare specimens of Dictyocha mandrai were observed in Samples 128-799A-lH-CC and 128799A-2H-CC. Except for the rare presence of Distephanus slavnicii in Section 128-799A-8H-CC (68.3 mbsf), for all practical purposes, the uppermost section down to Section 128-799A-11H-CC $(97.2$ mbsf) may be considered barren of these microfossils. Surprisingly, Distephanus octangulatus was absent from this hole.

In Sample 128-799A-11H-2,29 cm (89.29 mbsf), the joint occurrence of Dictyocha subarctios and Mesocena elliptica was recognized, suggesting that the top of the Dictyocha subarctios Zone is located between this sample and the overlying sample, $128-799 \mathrm{~A}-11 \mathrm{H}-1,115 \mathrm{~cm}(88.65$ $\mathrm{m})$, which in turn indicates the proximity of Brunhes/Matuyama Chron boundary. The Dictyocha subarctios Zone extends downward to Sample $128-799 \mathrm{~A}-15 \mathrm{H}-5,121 \mathrm{~cm}(133.3 \mathrm{mbs})$, where the underlying Pliocene Ammodochium rectangulare Zone was recognized by the presence of the named species. Sample 128-799A-17H-6, 83-85 cm (153.83 mbsf), is considered the top of the Ebriopsis antiqua antiqua Zone because of the LAD of the named species.

The next datum level recognized from Hole 799A was the highest occurrence of Distephanus jimlingii, which was observed in Section 128-799A-28X-CC (250.9 mbsf). According to the data from Site 798 , this datum level is placed near the top of Gauss Chron of approximately $2.6 \mathrm{Ma}$, which suggests an unusually high sedimentation rate near the end of Gauss Chron. 
Table 2. Distribution of silicoflagellates and ebridians from Hole 798B.

\begin{tabular}{|c|c|c|c|c|c|c|c|c|c|c|c|c|c|c|c|c|}
\hline $\begin{array}{l}\text { Core, } \\
\text { section, } \\
\text { interval } \\
(\mathrm{cm})\end{array}$ & 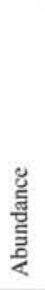 & 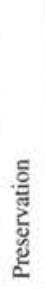 & 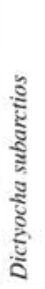 & 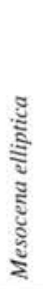 & 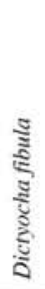 & 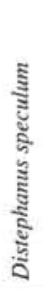 & 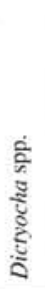 & 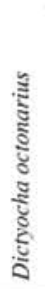 & 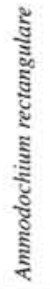 & 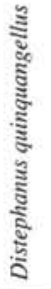 & 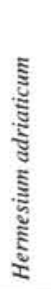 & 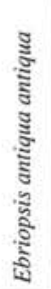 & 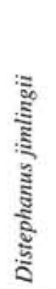 & 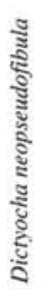 & 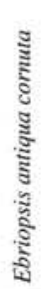 & 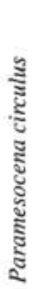 \\
\hline \multicolumn{17}{|l|}{ 128-798B- } \\
\hline $14 \mathrm{H}-1,28-30$ & F & $\mathrm{M}$ & $\mathrm{F}$ & $\mathbf{R}$ & . & . & & . & . & . & . & . & . & & . & . \\
\hline $14 \mathrm{H}-3,36-38$ & C & M & C & $\mathrm{R}$ & $\mathrm{R}$ & $\mathrm{R}$ & & . & & . & & & . & & . & . \\
\hline $14 \mathrm{H}-5,36-38$ & C & M & C & $\mathrm{R}$ & . & & & . & & & & & . & & . & \\
\hline $14 \mathrm{H}-7,36-38$ & $\mathrm{R}$ & M & F & $\mathrm{R}$ & $\mathrm{R}$ & $\mathrm{R}$ & $\mathrm{R}$ & & & . & & & . & & & \\
\hline $14 \mathrm{H}-\mathrm{CC}$ & $\mathrm{R}$ & M & $\mathrm{R}$ & & $\mathrm{R}$ & $\mathrm{R}$ & . & . & . & . & . & & . & & . & . \\
\hline $15 \mathrm{H}-\mathrm{CC}$ & C & M & C & . & $\mathrm{R}$ & $\mathrm{R}$ & $\mathrm{R}$ & $\mathrm{R}$ & . & . & . & . & . & . & & \\
\hline $16 \mathrm{H}-\mathrm{CC}$ & C & M & $\mathrm{R}$ & F & . & . & $\mathrm{F}$ & $\mathrm{R}$ & . & . & . & . & . & . & . & . \\
\hline $17 \mathrm{H}-\mathrm{CC}$ & c & M & F & . & F & $\mathrm{R}$ & $\mathrm{F}$ & . & . & . & . & . & . & . & & . \\
\hline $18 \mathrm{H}-\mathrm{CC}$ & F & M & F & . & . & . & $\mathrm{R}$ & . & . & . & . & . & . & . & . & . \\
\hline $19 \mathrm{X}-1,30-32$ & C & M & F & $\mathrm{F}$ & $\mathrm{R}$ & . & . & . & + & . & . & . & . & . & . & . \\
\hline $19 \mathrm{X}-3,30-32$ & C & M & F & R & F & . & $\mathrm{R}$ & . & . & & . & . & . & . & . & . \\
\hline $19 X-5,30-32$ & C & M & F & & $\mathrm{R}$ & $\mathbf{R}$ & & . & . & & & . & . & . & & . \\
\hline $19 \mathrm{H}-\mathrm{CC}$ & B & & & & & . & & . & . & & + & . & . & . & , & . \\
\hline $20 \mathrm{X}-1,30-32$ & F & M & . & & F & F & . & . & . & & . & & . & . & . & . \\
\hline $20 \mathrm{X}-3,30-32$ & $\mathrm{R}$ & M & $\mathrm{R}$ & . & & . & & & . & & . & & . & . & . & . \\
\hline $20 \mathrm{X}-6,30-32$ & A & M & $\mathrm{R}$ & $\mathrm{R}$ & A & . & & . & . & & . & & . & . & . & . \\
\hline $20 \mathrm{X}-8,30-32$ & A & M & . & & A & $\mathrm{R}$ & . & . & . & & . & & . & . & . & . \\
\hline 20X-CC & $\mathrm{R}$ & M & $\mathrm{R}$ & $\mathrm{R}$ & $\mathrm{R}$ & . & & . & . & & . & & . & . & & . \\
\hline $21 X-1,50-52$ & A & M & & $\mathrm{R}$ & F & A & $\mathrm{F}$ & . & $\mathrm{F}$ & & . & & . & . & . & . \\
\hline $21 X-3,50-52$ & A & $\mathrm{M}$ & & $\mathrm{R}$ & F & A & $\mathrm{R}$ & . & $\mathrm{F}$ & $\mathrm{F}$ & . & & . & . & . & . \\
\hline $21 X-5,50-52$ & A & M & . & C & $\mathrm{F}$ & . & . & . & C & C & . & . & . & . & . & . \\
\hline 21X-CC & c & M & . & $\mathrm{R}$ & . & C & . & . & . & & . & . & . & . & . & . \\
\hline $22 \mathrm{X}-\mathrm{CC}$ & B & . & & . & . & . & . & . & . & . & . & . & . & . & . & . \\
\hline $23 \mathrm{X}-1,30-32$ & C & $M$ & & . & F & C & . & . & $\mathrm{F}$ & . & . & & . & . & . & . \\
\hline $23 \mathrm{X}-2,32-34$ & B & . & & . & . & . & . & . & . & . & . & . & . & . & . & . \\
\hline $23 \mathrm{X}-3,30-325$ & A & M & . & $\mathrm{R}$ & C & A & $\mathrm{R}$ & . & $\mathrm{C}$ & . & & . & . & - & . & . \\
\hline $23 \mathrm{X}-5,98-100$ & B & & & & . & & & & & . & & . & & & . & . \\
\hline $23 \mathrm{X}-6,30-32$ & $\mathrm{~F}$ & M & & & $\mathrm{F}$ & F & & . & . & $\mathrm{F}$ & & . & . & . & . & . \\
\hline $23 \mathrm{X}-7,30-32$ & B & & & & & & & & & . & & . & & . & . & . \\
\hline $23 \mathrm{X}-8,38-40$ & B & & & . & & & & & & . & & . & & . & . & \\
\hline $23 \mathrm{X}-\mathrm{CC}$ & $\mathrm{R}$ & M & . & & . & $\mathrm{F}$ & & . & $\mathrm{R}$ & $\mathrm{F}$ & & . & & & & . \\
\hline $24 \mathrm{X}-1,33-35$ & B & & . & & & . & . & & . & . & & . & & . & . & . \\
\hline $24 \mathrm{X}-2,33-35$ & $\mathrm{R}$ & M & . & & $\mathrm{R}$ & $\mathrm{R}$ & . & . & . & . & & . & & . & & . \\
\hline $24 \mathrm{X}-4,33-35$ & $\mathrm{R}$ & M & . & & $\mathrm{R}$ & $\mathrm{R}$ & . & . & . & . & . & . & . & . & . & . \\
\hline $24 \mathrm{X}-5,24-26$ & $\mathrm{R}$ & $\mathrm{M}$ & . & & . & $\mathrm{R}$ & . & . & . & . & . & . & . & & . & . \\
\hline $24 \mathrm{X}-6,34-36$ & $\mathrm{R}$ & M & . & & . & $\mathrm{R}$ & & . & . & $\mathrm{R}$ & & . & & & . & . \\
\hline $24 \mathrm{X}-7,34-36$ & $\mathrm{~F}$ & M & . & & $\mathrm{R}$ & $\mathrm{F}$ & & & $\mathrm{R}$ & $\mathbf{R}$ & & . & . & . & . & . \\
\hline $24 \mathrm{X}, \mathrm{CC}$ & $\mathrm{R}$ & M & . & . & . & $\mathrm{R}$ & & . & $\mathrm{F}$ & . & & . & & . & & . \\
\hline $25 \mathrm{X}-\mathrm{CC}$ & $\mathrm{R}$ & $\mathrm{M}$ & . & & . & $\mathrm{R}$ & & . & $\mathrm{R}$ & $\mathrm{R}$ & . & . & . & . & . & . \\
\hline 26X-CC & C & M & . & & . & A & & . & F & & & & & & . & \\
\hline $27 \mathrm{X}-\mathrm{CC}$ & $\mathrm{R}$ & M & . & & $\mathrm{R}$ & $\mathrm{R}$ & & . & $\mathrm{R}$ & . & $\mathrm{R}$ & . & . & . & . & . \\
\hline $28 \mathrm{X}-\mathrm{CC}$ & $\mathrm{R}$ & M & . & & & $\mathrm{R}$ & , & . & . & . & & & . & . & . & \\
\hline 29X-CC & $\mathrm{R}$ & M & . & & & $\mathrm{R}$ & . & . & . & . & - & . & . & . & - & . \\
\hline $30 \times-3,30-32$ & B & & . & & & . & . & . & . & . & . & & . & & . & \\
\hline $30 \times-4,30-32$ & $\mathrm{R}$ & M & . & & & . & & . & . & . & . & $\mathrm{R}$ & . & . & . & . \\
\hline $30 X-5,30-32$ & F & M & . & . & & C & . & . & $\mathbf{R}$ & . & $\mathrm{R}$ & $\mathrm{R}$ & . & & . & . \\
\hline $30 \times-6,30-32$ & B & & . & & & . & . & . & . & . & & . & . & . & . & \\
\hline $30 X-6,75$ & B & & & & . & . & . & . & & . & & . & . & . & . & . \\
\hline $30 \mathrm{X}-\mathrm{CC}$ & $\mathrm{R}$ & M & . & & & $\mathrm{R}$ & . & . & . & . & & . & & & . & \\
\hline $31 X-1,30-32$ & A & G & . & & C & $\mathrm{R}$ & . & . & $\mathrm{F}$ & . & $\mathrm{R}$ & C & $\mathrm{F}$ & $\mathrm{R}$ & . & . \\
\hline $31 \mathrm{X}-2,34-36$ & C & $\mathrm{M}$ & . & & $\mathrm{F}$ & $\mathrm{R}$ & . & . & $\mathrm{F}$ & & . & $\mathrm{F}$ & $\mathrm{F}$ & $\mathrm{R}$ & & . \\
\hline $31 \times-4,30-32$ & C & M & . & & $\mathrm{F}$ & $\mathrm{R}$ & . & & A & . & . & $\mathrm{R}$ & $\mathrm{F}$ & $\mathrm{R}$ & . & . \\
\hline $31 X-5,30-32$ & $\mathrm{R}$ & M & . & & & $\mathrm{R}$ & & . & . & & . & . & . & . & $\mathrm{R}$ & . \\
\hline $31 \times-6,37-39$ & A & G & . & & $\mathrm{R}$ & C & . & . & C & & $\mathrm{R}$ & C & $\mathrm{R}$ & $\mathrm{R}$ & $\mathrm{R}$ & . \\
\hline $31 X-8,34-36$ & B & & . & & & . & . & . & . & . & . & . & . & . & . & . \\
\hline $31 \mathrm{X}-\mathrm{CC}$ & $\mathrm{R}$ & M & & & & $\mathrm{R}$ & & & $\mathrm{R}$ & & . & . & . & $\mathrm{R}$ & . & . \\
\hline $32 X-1,30-32$ & C & M & . & & $\mathrm{R}$ & . & . & . & . & & $\mathrm{R}$ & $\mathrm{F}$ & $\mathrm{R}$ & c & $\mathrm{R}$ & . \\
\hline $32 X-2,28-30$ & B & & . & & & . & . & . & . & & . & . & . & . & & . \\
\hline $32 X-3,30-32$ & C & M & . & & $\mathrm{R}$ & $\mathrm{R}$ & . & . & $\mathrm{R}$ & & $\mathrm{R}$ & $\mathrm{F}$ & . & C & $\mathrm{R}$ & . \\
\hline $32 X-4,30-32$ & B & & . & . & . & . & & . & . & & . & & . & . & & . \\
\hline
\end{tabular}

For explanation of abbreviations, see Table 1. 
Table 2 (continued).

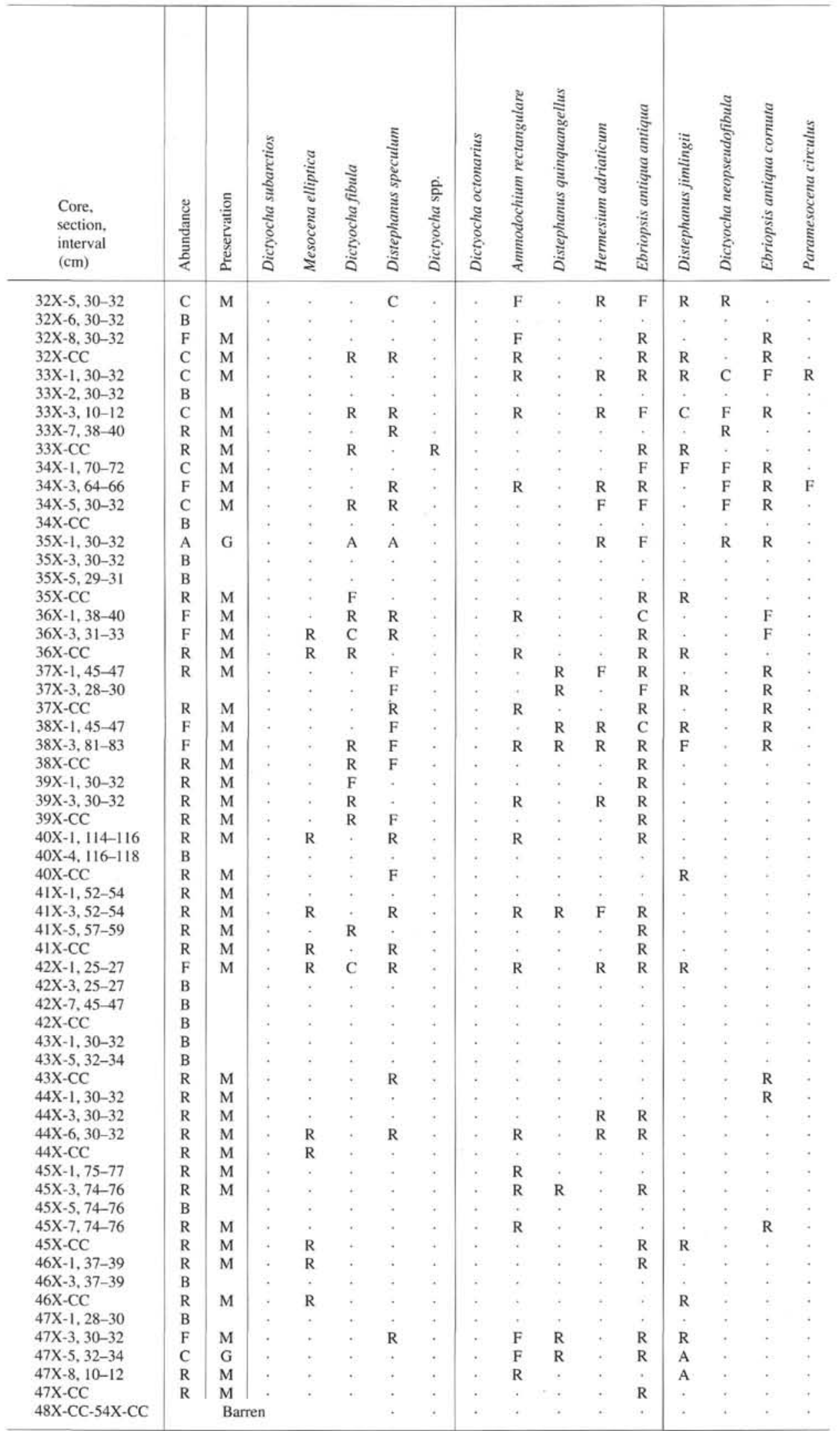


Table 3. Distribution of silicoflagellates and ebridians from Hole $798 \mathrm{C}$.

\begin{tabular}{|c|c|c|c|c|c|c|c|c|c|c|c|c|c|}
\hline $\begin{array}{l}\text { Core. } \\
\text { section, } \\
\text { interval } \\
\text { (cm) }\end{array}$ & 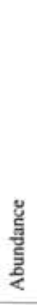 & 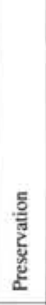 & 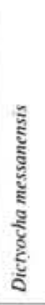 & $\begin{array}{l}\text { gू } \\
\text { हू } \\
\text { हू } \\
\frac{g}{\xi} \\
\text { हू }\end{array}$ & 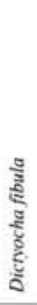 & 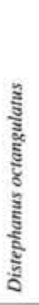 & 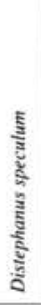 & 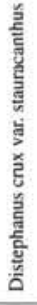 & 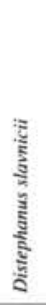 & 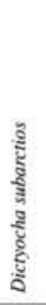 & 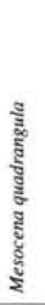 & 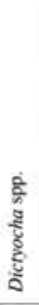 & 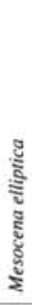 \\
\hline \multicolumn{14}{|l|}{$128-798 \mathrm{C}$. } \\
\hline $\mathrm{IH}-\mathrm{CC}$ & $\mathbf{R}$ & M & $\mathbf{R}$ & . & $\mathrm{R}$ & . & . & - & . & & - & . & . \\
\hline $2 \mathrm{H}-\mathrm{CC}$ & B & & t: & . & , & & . & & . & . & & . & \\
\hline $3 \mathrm{H}-\mathrm{CC}$ & $\mathbf{R}$ & M & $\mathbf{R}$ & & & $\mathbf{R}$ & - & , & , & . & & . & . \\
\hline $4 \mathrm{H}-\mathrm{CC}$ & $\mathbf{R}$ & M & $\therefore$ & & : & . & $\mathbf{R}$ & . & . & . & . & . & \\
\hline $5 \mathrm{H}-\mathrm{CC}$ & $\mathrm{F}$ & M & $\mathbf{R}$ & $\mathbf{R}$ & $\mathbf{R}$ & $\mathbf{R}$ & R & . & . & . & & . & . \\
\hline $6 \mathrm{H}-\mathrm{CC}$ & $\mathbf{R}$ & M & . & $\mathbf{R}$ & . & . & $\cdot$ & . & . & & . & . & . \\
\hline $7 \mathrm{H}-\mathrm{CC}$ & $\mathrm{R}$ & M & . & & : & i & $\mathbf{R}$ & $\mathbf{R}$ & . & . & - & . & . \\
\hline $8 \mathrm{H}-\mathrm{CC}$ & $\mathbf{R}$ & M & & & $\mathbf{R}$ & $\mathbf{R}$ & . & - & $\therefore$ & $\therefore$ & $\therefore$ & . & - \\
\hline $9 \mathrm{H}-1,20$ & $\mathrm{~F}$ & M & . & & . & . & . & $\mathrm{F}$ & $\mathbf{R}$ & $\mathbf{R}$ & $\mathbf{R}$ & . & . \\
\hline $9 \mathrm{H}-2,24$ & $\mathbf{R}$ & M & & , & . & . & . & $\mathbf{R}$ & $\mathbf{R}$ & $\mathbf{R}$ & & . & . \\
\hline $9 \mathrm{H}-4.30$ & $\mathrm{~F}$ & M & . & . & . & . & . & . & $\mathbf{R}$ & $\mathbf{R}$ & F & . & . \\
\hline $9 \mathrm{H}-5,125$ & A & M & . & . & . & - & . & $\mathrm{R}$ & A & . & . & . & - \\
\hline $9 \mathrm{H} \cdot 6,7$ & B & & & . & c. & & $\cdot$ & $\cdot$ & : & - & : & i. & . \\
\hline $9 \mathrm{H}-\mathrm{CC}$ & A & M & . & & $\mathbf{R}$ & . & & . & A & & A & $\mathbf{R}$ & . \\
\hline $10 \mathrm{H}-3.54$ & B & & . & . & 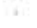 & . & $\cdot$ & . & : & s. & & . & \\
\hline $10 \mathrm{H}-5,105$ & $\mathrm{~F}$ & M & , & . & & . & . & . & $\mathbf{R}$ & $\mathrm{F}$ & . & . & . \\
\hline $10 \mathrm{H}-6.77$ & C & M & . & & . & . & & . & . & C & C & . & . \\
\hline 10H-CC & A & M & & & i & - & . & $\therefore$ & . & $\mathrm{F}$ & - & . & A \\
\hline $11 \mathrm{H}-\mathrm{CC}$ & C & M & . & & $\mathbf{R}$ & - & & $\mathbf{R}$ & . & C & $\therefore$ & $\therefore$ & F \\
\hline $12 \mathrm{H}-\mathrm{CC}$ & A & M & . & & $\mathbf{R}$ & . & . & - & . & C & $\mathbf{R}$ & $\mathbf{R}$ & F \\
\hline $13 \mathrm{H}-\mathrm{CC}$ & C & M & & & $\mathrm{F}$ & . & & . & . & $\mathbf{R}$ & . & . & F \\
\hline
\end{tabular}

For explanation of abbreviations, see Table 1 .

The Distephanus jimlingii Zone extends downward to at least Section 128-799A-35X-CC (316.9 mbsf) and is characterized by the continuous presence of the nominate taxon. Rare occurrences of the species were noted in Sections 128-799A-40X-CC (365.3 mbsf) and 128-799A-42X-CC (384.6 mbsf).

As stated earlier, the lower limit of this zone is defined by the initial appearance of $D$. jimlingii, which corresponds to the Miocene/Pliocene boundary of the region (Kobayashi, 1988).

Although Ammodochium rectangulare and Ebriopsis antiqua antiqua occur continuously down to Sample 128-799A-48X-CC (442.6 mbsf), no other age-diagnostic species were observed from the examined samples, and this group of siliceous microfossils was absent down to the bottom of this hole, Sample 128-799A-52X-CC (468.7 mbsf).

\section{TAXONOMIC NOTES}

Almost all the silicoflagellate and ebridian taxa encountered during the present analysis have been reported and adequately illustrated in various publications (Ling, 1971, 1973). Therefore, they are listed in alphabetical order according to the current nomenclature with the original references, and relevant remarks are made wherever necessary. Furthermore, illustrations are prepared for only the biostratigraphically important forms.

\section{Ebridians}

Ammodochium rectangulare (Schulz) Deflandre, 1932, pp. 303-305, figs. $1-13=$ Ebria antiqua var. rectangularis Schulz, 1928, p. 274, figs. 72 a-d. (Pl. 1, Fig. 11)

Ebriopsis antiqua antiqua Ling, 1977, p. 215, pl. 3, figs. 17, $18=$ Ebria antiqua Schulz, 1928 (partim), pp. 273, 274, fig. 69b (only) = Ebriopsis antiqua (Schulz) Ling, 1971 (partim), p. 693, pl. 2, figs. 3-5 (only). (PI. 1, Fig. 12)

Ebriopsis antiqua cornuta Ling, 1977, pp. 215-216, pl. 3, figs. 19-22 = Ebria antiqua Schulz, 1928 (partim), pp. 273, 274, figs. 69e and 69f (only) = Ebriopsis antiqua $($ Schulz), Ling 1971 (partim), p. 693, pl. 2, figs. 1, 2. (Pl. 1, fig. 13)
Hermesium adriaticum Zacharias, 1906, fide Loeblich et al., 1968, p. 168 , fig. 20 ; pl. 40, figs. 9 a-c, 10.

\section{Silicoflagellates}

Dictyocha fibula Ehrenberg, 1839, p. 129

Dictyocha mandrai Ling, 1977, pp. 209-210, pl. 1, figs. 13, 14 = Dictyocha fibula var. aculeata Lennermann, 1901, p. 261, pl. 11, figs. 1,2.

Dictyocha messanensis Haeckel, 1861, pp. 799, 800; for the synonymy and discussion, see Ling (1970, pp. 92, 93, pl. 18, fig. 14, as D. fibula var. messanensis (Haeckel)

Dictyocha neopseudofibula Kobayashi, 1988, pp. 59-60, pl. 5, figs. 1-9. (PI. 1, Fig. 1)

Dictyocha pentagona $($ Schulz $)=$ Dictyocha fibula var. pentagona Schulz, 1928 , p. 255 , figs. $41 \mathrm{a}, 4 \mathrm{lb}$.

Dictyocha subarctios Ling, 1970, pp. 95, 96, pl. 18, figs. 16-18, pl. 19, figs. 1-4.

Dictyocha spp.

Remarks: Due to their sporadic occurrence and low abundance, specimens referable to $D$. aspera aspera Bukry, $D$. ausonia Deflandre, $D$. brevispina (Lemmermann) Bukry, D. calida calida Poelchau, and D. delicaae Bukry are combined under the present taxon during the present study.

Distephanus crux var. stauracanthus Ling (not Ehrenberg), 1970, p. 96, pl. 19, figs. 7,$8 ; 1973$, p. 753 , pl. 1, figs. 20,$21 ; 1975$, p. 772 , pl. 1, figs. 25 , 26. (PI. 1, Fig. 3)

Remarks: The specimens originally observed from the central North Pacific and subsequently recognized through the North Pacific by the present author are different from Distephanus stauracanthus (Ehrenberg) in that the basal ring definitely shows eight sides, although it is still close to square in shape, and that all eight radial spines are short and nearly the same length, although the four at the square comers are slightly longer than the remaining four. A square-shaped apical structure is much smaller than that of the basal ring. Apparently, this species has a short geological range: occurring jointly with $D$. slavnicii approximately at or slightly earlier than the LAD of Dictyocha subarctios and disappearing soon after the extinction of D. subarctios in the North Pacific and the Sea of Japan. Therefore, there is a good possibility that, upon further detailed study, the LAD of this species may serve as a useful datum level.

Distephanus jimlingii (Bukry) Bukry, 1979, p. 561, pl. e, figs. 7-12=D. boliviensis jimlingii Bukry, 1975, p. 688, pl. 1, figs. 6. 7. (Pl.1, Fig. 4)

Distephanus octangulatus Wailes, 1932, p. 216, fig. $3=$ D. speculum var. octonarius Ehrenberg forma Wailes, 1928, p. 14, pl. 12, fig. 33. (Pl. 1, Fig. 5)

Distephanus quinguangellus Bukry and Foster, 1973, p. $828=$ D. speculum var. pentagonus Lemmermann, 1901, p. 264, pl. I1, fig. 19.

Distephanus slavnicii (Jerković), Bukry, 1973b, pl. 2, figs. 1-2 = D slavnici slavnicii Jerković, 1965, p. 124, pl. 1, figs. 18, 19; pl. 2 = Distephanus sp. cf. D. slavnicii slavnicii (Jerković), Ling, 1971, p. 692, pl. 1, figs. 10, 11. (Pl. 1, Fig. 6)

Distephanus speculum (Ehrenberg) Haeckel, 1887, p. $1565=$ Dictyocha speculum Ehrenberg, 1839, p. 129. (PI. 1, Fig. 7)

Remarks: In addition to the typical form, specimens with multiple apical windows but the similar size range are also included under the present taxon.

Mesocena elliptica (Ehrenberg) Ehrenberg, 1844, p. 71, $84=$ Dictyocha (Mesocena) elliptica Ehrenberg, 1840, p. 208. (Pl. 1, Fig. 8)

Mesocena quadrangula Ehrenberg ex Haeckel, 1887, p. 1556. (PI. 1, Fig. 9) 
Paramesocena circulus (Ehrenberg) Locker and Martini, 1986, p. 909, pl. 9, figs. 2-4, pl. 12, figs. 4, 5. = Dictyocha (Mesocena) circulus Ehrenberg, 1840, p. 208. (Pl. 1, Fig. 10)

\section{ACKNOWLEDGMENTS}

The author acknowledges financial support from the National Science Foundation through the Texas A\&M Research Foundation, which enabled him to participate on the cruise and to carry out the post-cruise analysis. Samples from Leg 127 were made available by shipboard scientists upon approval of the ODP curatorial office. Special thanks are due to Drs. Joyce R. Blueford, Kevin McCartney, Stanley A. Kling, and Kozo Takahashi for reviewing the manuscript, Muhong Chen, Mohammad Fariduddin, and Lisa Paulson for technical assistance during the preparation of the manuscript, the Leg 128 shipboard colleagues for stimulating discussion, and the personnel of Leg 128 on the JOIDES Resolution (SEDCO/BP 471). Without their efforts, these submarine sediments would never have appeared above sea level.

\section{REFERENCES}

Barron, J. A., 1980. Lower Miocene to Quaternary diatom biostratigraphy of Leg 57, off northeastern Japan, Deep Sea Drilling Project. In von Huene, R., Nasu, N., et al., Init. Repts. DSDP, 56, 57 (Pt. 2): Washington (U.S. Govt. Printing Office), 641-685.

Bukry, D., 1973a. Coccolith and silicoflagellate stratigraphy, Deep Sea Drilling Project Leg 18, eastern North Pacific. In Kulm, L. D., von Huene, R., et al., Init. Repts. DSDP, 18: Washington (U.S. Govt. Printing Office), 817-831.

- 1973b. Coccoliths and silicoflagellates from Deep Sea Drillin Project Leg 19, North Pacific Ocean and Bering Sea. In Creager, J. S., Scholl, D. W., et al., Init. Repts. DSDP, 19: Washington (U.S. Govt. Printing Office), 857-867.

1975. Coccolith and silicoflagellate stratigraphy, northwestern Pacific Ocean, Deep Sea Drilling Project Leg 32. In Larson, R. L., Moberly, R., et al., Init. Repts. DSDP, 32: Washington (U.S. Govt. Printing Office), 677-701.

1979. Coccolith and silicoflagellate stratigraphy, northern Mid-Atlantic Ridge and Reykjanes Ridge, Deep Sea Drilling Project Leg 49. In Luyendyk, B. P., Cann, J. R., et al., Init. Repts. DSDP, 49: Washington (U.S. Govt. Printing Office), 551-581.

Bukry, D., and Foster, J. H., 1973. Silicoflagellate and diatom stratigraphy, Leg 16, Deep Sea Drilling Project. In Van Andel, T. H., Heath, G. R., et al., Init. Repts. DSDP, 16: Washington (U.S. Govt. Printing Office), 815-871.

Bukry, D., and Monechi, S., 1985. Late Cenozoic silicoflagellates from the Northwest Pacific, Deep Sea Drilling Project Leg 86: paleotemperature trends and texture classification. In Heath, G. R., Burckle, L. H., et al., Init. Repts. DSDP, 86: Washington (U.S. Govt. Printing Office), 367-397.

Deflandre, G., 1932. Remarques sur quelques ébriacées. Bull. Soc. Zool. Fr, $57: 302-315$.

Dumitrică, P., 1967. Dictyocha bachmanni n. sp. et considérations sur la lignée phylogénetique Dictyocha crux-D. saturacantha-D. bachmanni. Arch. orig. Centre Document, C.N.R.S., Cah. Micropaleontol., Ser. 1, 4:1-6.

Ehrenberg, C. G., 1839. Über die Bildung der Kreidefelsen und des Kreidemergels durch unsichtbare Organismen. Abh. K. Akad. Wiss. Berlin, 1838:59-148.

$\longrightarrow$, 1840.274 Blätter von ihm selbst ausgeführter Zeichnunmgen von ebenso vielen Arten. K. Preuss. Akad. Wiss. Berlin, Ber., Jahrg. 1840:197-219.

, 1844. Mittheilung über zwei neue Lager von Gebirgsmassen aus Infusorien als Meeres Absazt in Nord-Amerika und eine Vergleichung derselben mit den organischen Kreide-Gebilded in Europa und Afrika. K. Preuss. Akad. Wiss. Berlin Ber., Jahrg. 1844:57-97.

1845. Neue Untersuchungen über das kleinste Leben als geologisches Moment. Mit kurzer Charakteristik von 10 neuen Genera und 66 neuen Arten. Verb. K. Preuss. Akad. Wiss. Berlin Ber., Jahrg. 1845:53-87. , 1854. Mikrogeologie, das Erden und Felsen schaffende Wirken des unsichtbar kleinen selbstandigen Lebens auf der Erde: Leipzig (Leopold Voss), 1-374.

Gran, H. H., and Braarud, T., 1935. A quantitative study of the phytoplankton in the Bay of Fundy and the Gulf of Maine (including observations of hydrography, chemistry and turbidity). Can. J. Fish. Aquat. Sci., 1:279-467.
Haeckel, E., 1861. Über neue, lebende Radiolarien des Mittelmeeres. K. Preuss. Akad. Wiss. Berlin, Monatsber., Jahrg. 1860:794-817. 1887. Report on the Radiolaria collected by H.M.S. Challenger during the years 1873-1876. Rep. Sci. Results Voy. H.M.S. Challenger, Zool., 18 .

Hays, J. D., Saito, T., Opdyke, N. D., Burckle, L. H., 1969. Pliocene-Pleistocene sediments of the Equatorial Pacific: their paleomagnetic, biostratigraphic, and climatic record. Geol. Soc. Am. Bull., 80:1481-1514.

Ingle, J. C., Jr., Suyehiro, K., von Breymann, M. T., 1990. Proc. ODP, Init. Repts., 128: College Station, TX (Ocean Drilling Program).

Jerković, L., 1965. Sur quelques silicoflagellides de Yougoslavie. Rev. Micropaleontol., 8:121-130.

Kobayashi, H., 1988. Neogene silicoflagellate biostratigraphy of the Japan Sea Coastal region, with reference to DSDP Hole 438A. Sci Rep. Tohoku Univ., Ser. 2 (Geology), 59:1-98.

Koizumi, I., 1975. Neogene diatoms from the western margin of the Pacific Ocean, Leg 31, Deep Sea Drilling Project. In Karig, D. E., Ingle, J. C. Jr., et al., Init. Repts. DSDP, 31: Washington (U.S. Govt. Printing Office), 779-819.

Koizumi, I., and Tanimura, Y., 1985. Neogene diatom biostratigraphy of the middle latitude western North Pacific, Deep Sea Drilling Project Leg 86. In Heath, G. R., Burckle, L. H., et al., Init. Repts. DSDP, 86: Washington (U.S. Govt. Printing Office), 269-300.

Lemmermann, E., 1901. Silicoflagellatae. Ber. Dtsch. Bot. Ges., Jahrg. 19:247-271.

Ling, H. Y., 1970. Silicoflagellates from Central North Pacific core sediments. Bull. Am. Paleontol., 58:85-129.

- 1971. Silicoflagellates and ebridians from the Shinzan Diatomaceous Mudstone member of the Onnagawa Formation (Miocene), Northeast Japan. In Farinacci, A. (Ed.), Proc. 2nd Planktonic Conf. Roma: Rome (Ed. Technosci.), 689-703.

, 1973. Silicoflagellates and ebridians from Leg 19. In Creager, J. S., Scholl, D. W., et al., Init. Repts. DSDP, 19: Washington (U.S. Govt. Printing Office), 751-775.

, 1975. Silicoflagellates and ebridians from Leg 31. In Karig, D. E., Ingle, J. C., Jr., et al., Init. Repts. DSDP, 31: Washington (U.S. Govt. Printing Office), 763-777.

, 1976. Distribution and biostratigraphic significance of Dictyocha subarctics (silicoflagellate) in the North Pacific. Trans. Proc. Palaeontol. Soc. Jpn., 101:264-270.

, 1977. Late Cenozoic silicoflagellates and ebridians from the eastern North Pacific region. In Saito, T., and Ujiie, H. (Eds.), Proc. First Internat. Congr. Pacific Neogene Stratigraphy, Tokyo 1976, 205-233.

, 1980. Silicoflagellates and ebridians from Leg 55. In Jackson, E. D., Koizumi, I., et al., Init. Repts. DSDP, 55: Washington (U.S. Govt. Printing Office), 375-385.

Locker, S., and Martini, E., 1986. Silicoflagellates and some sponge spicules from the southwest Pacific, Deep Sea Drilling Project, Leg 90. In Kennett, J. P., von der Borch, C. C., et al., Init. Repts. DSDP, 90: Washington (U.S. Govt. Printing Office), 887-924.

Loeblich, A. R., III, Loeblich, L. A., Tappan, H., and Loeblich, A. R., Jr., 1968. Annotated index of fossil and Recent silicoflagellates and ebridians with descriptions and illustrations of validly proposed taxa. Geol. Soc. Am. Mem., 106:xi, 319.

Saito, T., and Burckle, L. H., 1977. Occurrence of silicoflagellate Mesocena elliptics: further evidence on the age of the Wakimoto Formation, Oga Peninsula, Japan and the recognition of the Jaramillo event. J. Geol. Soc. Jpn., 83:181-186.

Schulz, P., 1928. Beitrage zur Kenntnis fossiler und rezenter Silicoflagellaten. Bot. Arch., 21:225-292.

Wailes, G. H., 1928. Fresh-water and marine Protozoa from British Columbia with description of new species. Vancouver Mus. Notes, 3:9-14.

, 1932. Description of new species of Protozoa from British Columbia. Can. Biol. Fish. Contrib., 7:215-219.

Zacharias, O., 1906. Eine neue Dictyochide aus dem Mittelmeer, Hermesinum adriaticum n. g., n. sp. Arch. Hydrobiol. u. Planktonk., 1:394-398.

Date of initial receipt: 22 May 1991

Date of acceptance: 20 August 1991

Ms 127/128B-126 
Table 4. Distribution of silicoflagellates and ebridians from Hole 799A.

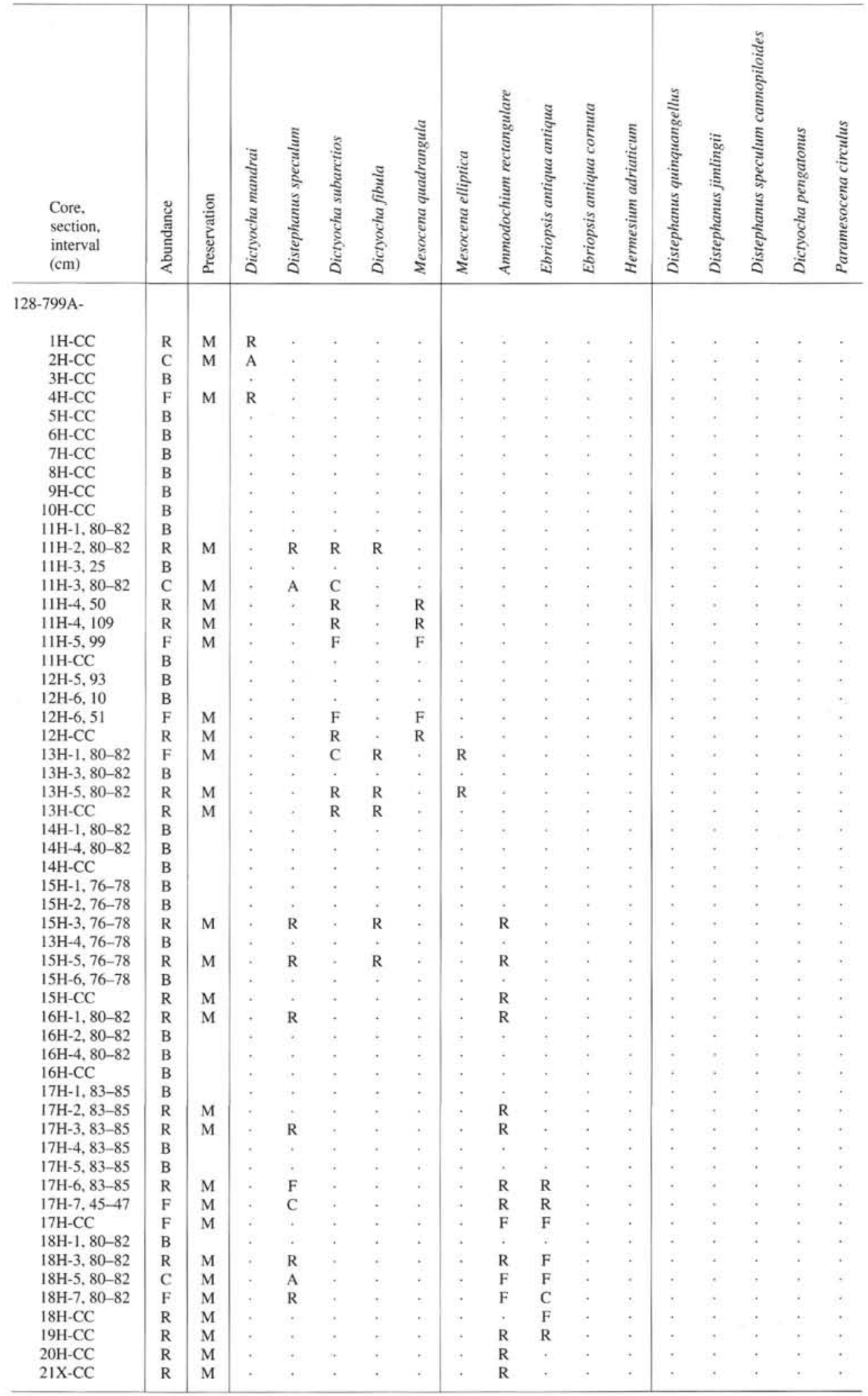

For explanation of abbreviations, see Table 1. 
Table 4 (continued).

\begin{tabular}{|c|c|c|c|c|c|c|c|c|c|c|c|c|c|c|c|c|c|}
\hline $\begin{array}{l}\text { Core, } \\
\text { section, } \\
\text { interval } \\
(\mathrm{cm})\end{array}$ & 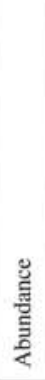 & 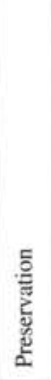 & 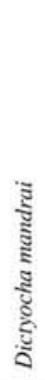 & 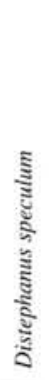 & 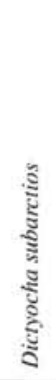 & 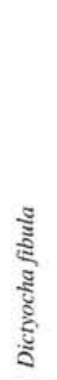 & 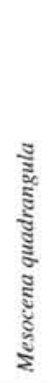 & 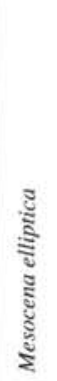 & 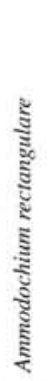 & 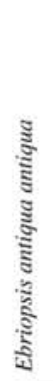 & 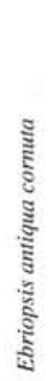 & 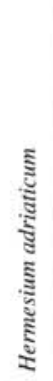 & 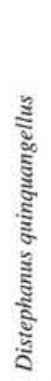 & 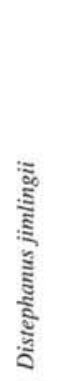 & 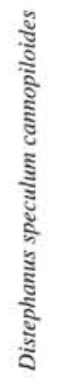 & 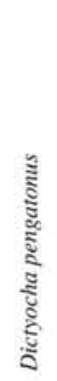 & 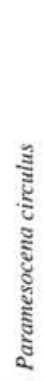 \\
\hline $22 \mathrm{X}-\mathrm{CC}$ & $\mathrm{R}$ & M & & . & & . & . & . & . & . & $\mathrm{R}$ & & . & . & . & . & . \\
\hline 23X-CC & $\mathrm{R}$ & M & & . & & . & . & . & $\mathrm{R}$ & $\mathrm{R}$ & . & & . & . & . & . & \\
\hline $24 \mathrm{X}-\mathrm{CC}$ & $\mathrm{R}$ & M & & . & & . & . & . & $\mathrm{R}$ & . & . & . & . & . & . & . & . \\
\hline $25 \mathrm{X}-1,80-82$ & B & & & . & & & & & & & & . & . & . & . & . & . \\
\hline $25 X-3,80-82$ & B & & & . & . & & & & & & & . & . & . & & . & . \\
\hline $25 X-5,79-81$ & F & M & . & $\mathrm{R}$ & . & & & & F & F & & $\mathrm{R}$ & . & & . & . & \\
\hline $25 \mathrm{X}-\mathrm{CC}$ & $\mathrm{R}$ & M & . & & . & & & & $\mathrm{R}$ & . & & . & . & . & . & . & \\
\hline $27 X-1,80-82$ & F & M & . & $\mathrm{R}$ & . & & . & & $\mathrm{R}$ & $\mathrm{R}$ & & $\mathrm{R}$ & $\mathrm{R}$ & . & & . & \\
\hline $27 \mathrm{X}-3,80-82$ & $\mathrm{R}$ & M & . & $\mathrm{R}$ & . & & . & $\mathrm{R}$ & . & . & & . & $\mathrm{R}$ & . & . & . & \\
\hline $27 \mathrm{X}-\mathrm{CC}$ & $\mathrm{R}$ & M & . & . & . & & & & . & $\mathrm{R}$ & & . & . & . & . & . & \\
\hline $28 \mathrm{X}-1,44-46$ & $\mathrm{R}$ & M & . & . & . & & & & $\mathrm{F}$ & $\mathrm{R}$ & & . & $\mathrm{R}$ & . & . & . & \\
\hline $28 \mathrm{X}-\mathrm{CC}$ & F & M & . & . & & & & $\mathrm{R}$ & & F & $\mathrm{R}$ & . & . & . & . & . & \\
\hline $29 \mathrm{X}-1,80-82$ & F & M & & $\mathrm{R}$ & & & & & $\mathrm{C}$ & $\mathrm{R}$ & F & . & . & . & . & . & \\
\hline $29 X-3,80-82$ & B & & & . & & & & & & . & & & . & . & & . & \\
\hline $29 \times-5,60-62$ & A & G & . & $\mathrm{R}$ & & & . & $\mathrm{F}$ & A & C & $\mathrm{C}$ & $\mathrm{R}$ & $\mathrm{R}$ & F & . & . & \\
\hline 29X-CC & $\mathrm{R}$ & M & & & . & . & & $\mathrm{R}$ & $\mathrm{R}$ & $\mathrm{R}$ & . & . & . & $\mathrm{R}$ & . & . & . \\
\hline $30 X-1,80-82$ & $\mathrm{R}$ & M & & & . & . & . & $\mathrm{R}$ & F & $\mathrm{R}$ & $\mathrm{R}$ & . & . & $\mathrm{R}$ & $\mathrm{R}$ & & . \\
\hline $30 \mathrm{X}-3,80-82$ & $\mathrm{R}$ & M & . & & . & . & . & . & $\mathbf{R}$ & . & $\mathrm{R}$ & . & . & $\mathrm{R}$ & $\mathrm{R}$ & & . \\
\hline $30 \mathrm{X}-\mathrm{CC}$ & $\mathrm{R}$ & M & . & & . & . & . & $\mathbf{R}$ & $\mathrm{R}$ & $\mathrm{R}$ & $\mathrm{R}$ & . & & $\mathrm{R}$ & . & & \\
\hline $31 X-1,80-82$ & $\mathrm{R}$ & M & , & & & . & & . & $\mathrm{R}$ & $\mathrm{R}$ & $\mathrm{R}$ & & . & $\mathrm{R}$ & & $\mathrm{R}$ & . \\
\hline $31 X-3,80-82$ & $\mathrm{R}$ & M & . & . & & . & & . & $\mathrm{F}$ & . & $\mathrm{R}$ & & . & $\mathrm{R}$ & . & $\mathrm{R}$ & . \\
\hline $31 X-5,80-82$ & C & M & & & & . & & . & C & . & $\mathrm{R}$ & & . & $\mathrm{F}$ & . & $\mathrm{R}$ & . \\
\hline $31 \mathrm{X}-\mathrm{CC}$ & $\mathrm{R}$ & M & & . & & . & & . & . & $\mathrm{R}$ & $\mathrm{R}$ & & . & $\mathrm{R}$ & . & & . \\
\hline $32 X-1,80-82$ & A & G & & & & . & & . & A & . & $\mathrm{R}$ & & . & A & $\mathrm{R}$ & F & . \\
\hline $32 X-3,80-82$ & C & M & & F & & . & & . & C & $\mathrm{R}$ & . & & . & C & . & $\mathrm{R}$ & \\
\hline $32 X-5,80-82$ & C & M & & $\mathrm{R}$ & & . & & . & C & $\mathrm{R}$ & . & & . & $\mathrm{F}$ & . & & . \\
\hline $32 \mathrm{X}-\mathrm{CC}$ & $\mathrm{R}$ & M & . & . & & & & & $\mathrm{R}$ & $\mathrm{R}$ & & & . & $\mathrm{R}$ & . & . & . \\
\hline $33 \mathrm{X}-\mathrm{CC}$ & $\mathrm{R}$ & M & & . & . & & . & & R & $\mathrm{R}$ & & . & . & $\ddot{R}$ & . & . & \\
\hline $34 \mathrm{X}-\mathrm{CC}$ & $\mathrm{R}$ & M & . & . & & & & & F & $\mathrm{R}$ & $\mathrm{R}$ & . & . & $\mathrm{R}$ & . & . & \\
\hline $35 \mathrm{X}-\mathrm{CC}$ & $\mathrm{R}$ & M & . & . & . & & . & & R & R & $\mathrm{R}$ & & & $\mathrm{R}$ & . & $\mathrm{R}$ & \\
\hline $36 \mathrm{X}-\mathrm{CC}$ & B & & . & & . & & . & & . & . & . & . & & . & . & . & . \\
\hline $37 \mathrm{X}-\mathrm{CC}$ & $\mathrm{R}$ & M & . & & . & & . & . & $\mathrm{R}$ & $\mathrm{R}$ & $\mathrm{R}$ & . & & & . & . & \\
\hline $38 \mathrm{X}-\mathrm{CC}$ & R & M & . & & . & . & . & . & . & $\mathrm{R}$ & . & . & & . & & $\mathrm{R}$ & . \\
\hline 39X-CC & B & & . & & . & & . & . & . & ?. & . & . & & . & . & . & . \\
\hline $40 \mathrm{X}-1,86-88$ & C & M & . & & . & & & . & F & F & $\mathrm{F}$ & $\mathrm{F}$ & . & . & . & & . \\
\hline $40 X-3,86-88$ & $\mathrm{R}$ & M & . & & & . & & . & & $\mathrm{R}$ & $\mathrm{R}$ & & & . & . & & . \\
\hline $40 X-5,86-88$ & $\mathrm{R}$ & M & . & & . & . & & . & $\mathrm{R}$ & $\hat{R}$ & ? & $\mathrm{R}$ & & $\mathrm{R}$ & . & & . \\
\hline $40 \mathrm{X}-\mathrm{CC}$ & $\mathrm{F}$ & M & . & & . & . & & . & F & $\mathrm{F}$ & $\mathrm{R}$ & & . & $\mathrm{F}$ & & . & . \\
\hline $41 X-1,80-82$ & $\mathrm{~F}$ & M & . & . & & . & & . & $\mathrm{R}$ & $\mathrm{R}$ & . & $\mathrm{F}$ & . & $\mathrm{F}$ & . & . & \\
\hline $41 X-3,80-82$ & $\mathrm{R}$ & M & . & . & & . & & . & $\mathrm{R}$ & $\mathrm{R}$ & F & . & $\mathrm{R}$ & $\mathrm{R}$ & . & . & . \\
\hline $41 X-5,80-82$ & C & G & & $\mathrm{R}$ & & . & & . & C & F & F & & $\mathrm{R}$ & $\mathrm{R}$ & . & . & \\
\hline $41 \mathrm{X}-\mathrm{CC}$ & $\mathrm{R}$ & $\mathrm{M}$ & . & . & . & . & . & . & $\mathrm{R}$ & $:$ & : & . & . & . & . & . & . \\
\hline $42 \mathrm{X}-\mathrm{CC}$ & $\mathrm{R}$ & M & . & . & . & . & & . & $\mathrm{R}$ & $\mathbf{R}$ & & & & $\mathrm{R}$ & . & . & . \\
\hline 43X-CC & $\mathrm{R}$ & $\mathrm{M}$ & . & . & . & . & & . & $\mathrm{R}$ & . & & & & . & . & . & . \\
\hline 44X-CC & B & & $\therefore$ & . & . & . & & . & & . & & & . & . & & . & . \\
\hline $45 \mathrm{X}-\mathrm{CC}$ & $\mathrm{R}$ & $\mathrm{M}$ & . & . & & . & & . & $\mathbf{R}$ & $\mathrm{R}$ & & & & & . & . & \\
\hline 46X-CC & $\mathrm{R}$ & M & . & . & . & . & . & . & $\mathrm{R}$ & $\mathrm{R}$ & . & & & & & . & . \\
\hline $47 \mathrm{X}-\mathrm{CC}$ & $\mathrm{R}$ & M & . & . & . & . & . & . & $\mathrm{R}$ & $\mathrm{R}$ & . & & . & & . & . & \\
\hline $48 \mathrm{X}-1,79-81$ & $\mathrm{R}$ & M & . & . & . & & . & & . & $\mathrm{R}$ & . & & . & . & . & . & . \\
\hline $48 X-3,79-81$ & $\mathrm{R}$ & M & . & . & . & & . & & $\mathrm{R}$ & $\mathrm{R}$ & & . & . & $\mathrm{R}$ & . & . & \\
\hline 48X-CC & $\mathrm{R}$ & M & . & . & . & & . & & $\mathrm{R}$ & $\mathrm{R}$ & & . & . & . & . & . & . \\
\hline $49 \mathrm{X}-1,90-92$ & $\mathbf{R}$ & M & . & . & . & & & & F & $\mathrm{R}$ & & . & . & & . & . & . \\
\hline 49X-CC & $\mathrm{R}$ & M & . & . & . & . & . & . & . & . & . & . & . & . & . & . & $\mathrm{R}$ \\
\hline $50 X-1,81-83$ & $\mathrm{~F}$ & M & . & & & . & . & . & C & $\mathrm{R}$ & $\mathrm{R}$ & $\mathrm{R}$ & & & . & & . \\
\hline $50 X-2,93-95$ & $\mathrm{~F}$ & M & . & . & . & . & . & . & C & $\mathrm{F}$ & $\mathrm{R}$ & . & . & & . & & $\mathrm{R}$ \\
\hline $50 X-3,81-83$ & $\mathrm{~F}$ & M & . & . & . & . & . & . & F & $\mathbf{R}$ & $\mathrm{R}$ & $\mathrm{R}$ & . & . & . & . & $\mathrm{R}$ \\
\hline $50 \mathrm{X}-\mathrm{CC}$ & B & & . & . & & . & . & . & . & & . & . & . & . & . & . & . \\
\hline 5IX-CC & B & & . & . & . & . & . & & . & . & . & . & . & & . & . & . \\
\hline
\end{tabular}




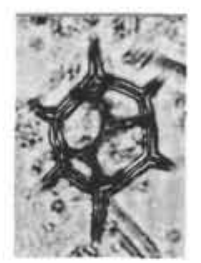

1

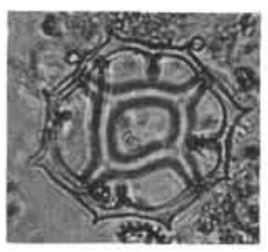

5

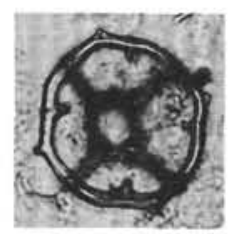

6

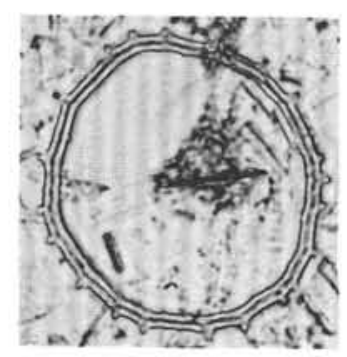

10

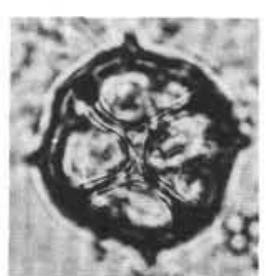

2

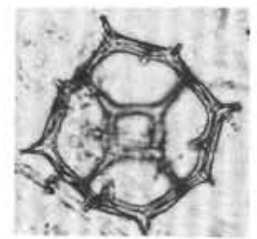

3

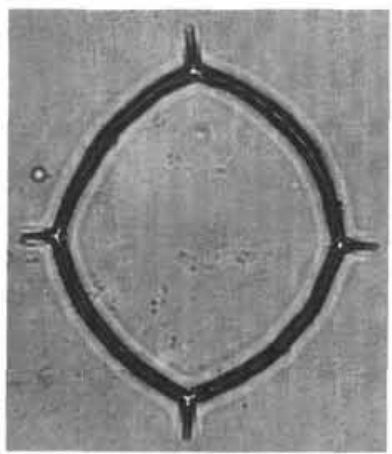

8

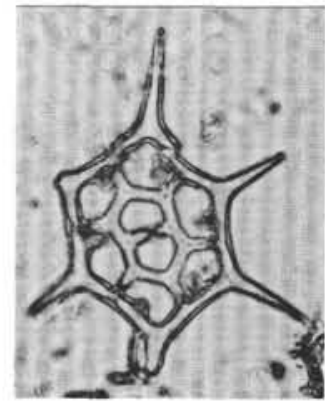

4

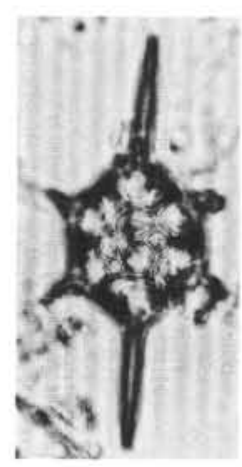

7

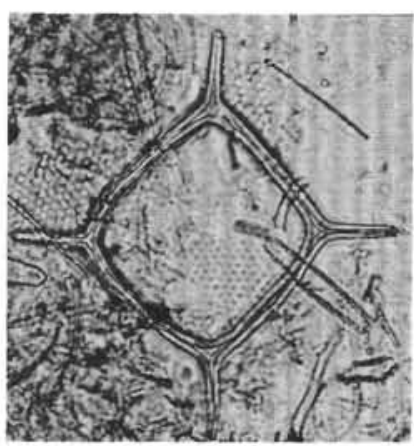

9

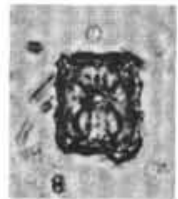

11

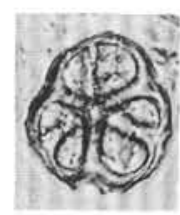

12

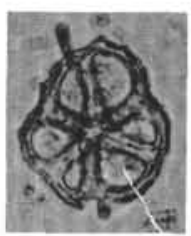

13

Plate 1. 1. Dictyocha neopseudofibula, Sample 128-798B-32-1,32-34 cm, L-2 (GI3/4). 2. Dictyocha subarctios, Section 128-798A-15H-CC, L-2 (V33/3). 3. Distephanus crux var. stauracanthus, Sample 128-798A-6H-7, 20-22 cm, R-1 (Z5/2). (Mil/2). 5. Distephanus octangulatus. Sample 128-798A-8H-2, 30-32 cm, L-2 (K6/2).

4. Distephanus jimlingii, Sample 128-798B-47X-5, $32-34 \mathrm{~cm}, \mathrm{~L}-2$ (Y27/0). 7. Distephanus speculum, Sample 128-799A-32X-1, 80-82 cm, L-2 (Y9/2). 8. Mesocena elliptica, Sample 128-799A-29X-5, 60-62 cm, L-2 (EI5/2). 9. Mesocena quadrangula, Sample 128-798A-15H, CC, L-2 (U22/4). 10. Paramesocena circulus, Sample 128-798B-32X-1, 32-34 cm, L-2 (GI7/3). 11. Ammodochium rectangulare, Sample 128-798B-47X-5, 32-34 cm, L-2 (K8/1). 12. Ebriopsis antiqua antiqua, Sample 128-799A-18H-CC, L-2 (C22/4). 13. Ebriopsis antiqua cornuta, Sample 128-798B-47X-5, 32-34 cm, L-2 (SI5/3). All magnification $=500 \times$. 